REVIEW

\title{
How to make the RCPCH CPD guidelines work for you
}

\section{A Thomson}

Arch Dis Child 2006;91:65-67. doi: 10.1136/adc.2005.075085

Continuing professional development (CPD) is defined as a systematic process of lifelong learning and professional development. Its aim is to enable career grade doctors (consultants, associate specialists, staff grades, and their equivalents) to maintain and enhance their knowledge, skills, and competence for effective clinical practice to meet the needs of children. This article is designed to assist and improve acquisition of CPD.

$\mathrm{T}$ here has been an evolution in thinking about continuing professional development (CPD) over the last decade, with a shift away from passive learning at approved events in favour of interactive learning structured to meet the learning needs set out in an individual's professional development plan (PDP) that has been agreed at appraisal. The Royal College of Paediatrics and Child Health (RCPCH) scheme has evolved to accommodate this philosophy, but retains a commitment to collection of evidence. The General Medical Council, in its autumn 2004 consultation on revalidation, reminded the profession that it advised doctors to collect evidence of learning from 2002. ${ }^{1}$ This evidence is also essential for annual appraisal.

\section{IS THERE A PROBLEM?}

The RCPCH audits 5\% of the CPD scheme participants each year. The first two annual audits $^{2}$ showed that paediatricians are able to use the RCPCH CPD guidelines as the basis of their annual CPD. In particular, most can acquire at least 25 external CPD points each year and can present evidence of these, on request. However, a significant minority of paediatricians do not claim an adequate number of points or cannot produce evidence. This is individually and corporately risky, potentially allowing professional skills, clinical practice, and standards of teaching and training to decline. It also opens up potential clinical governance, risk management. and medico-legal issues.

\section{THE RCPCH SCHEME IN OUTLINE}

CPD is a professional obligation for all doctors. Without CPD doctors will not be able to undertake annual appraisal, nor will they be able to proceed to revalidation. ${ }^{3}$ To assist paediatricians, the RCPCH issues guidelines on CPD annually. ${ }^{4}$ The guidelines conform to the " 10 Principles of $\mathrm{CPD}^{\prime \prime}$ from the Academy of Medical Royal Colleges $^{5}$ and contain a list of continuing education activities that qualify as CPD. The core of this advice stays constant. Major variation in CPD advice would create instability. Some paediatricians may prefer to participate in other CPD schemes, but should ensure any alternative demonstrably meets their needs.

The model for CPD in paediatrics and child health is intended to be practical and realistic. The RCPCH CPD guidance is phrased to allow some flexibility in practice. A range of CPD activities needs to be selected, to achieve a balance between broad based and specialist learning to suit individual professional needs. Usually, CPD should be approximately equally divided between internal and external activities over a five year period. External CPD is defined as education involving interaction with colleagues outside the individual's own department. It usually requires study leave in protected time. It includes organised meetings and courses, as well as personal visits to outside institutions to acquire new skills. On average, paediatricians should claim a minimum of 25 external CPD credits each year. Internal CPD includes hospital and community unit clinical meetings, those involving multidisciplinary participation, grand rounds, and clinical audit meetings. It does not require study leave. There is no lower limit set to the number of sessions of internal CPD, only a lower limit on the percentage of credits overall that should come from external CPD.

The sub-categories-clinical, academic, professional-should also be used, with about $80 \%$ of CPD credits typically being in the clinical category. Clinical educational activities relate to the learning and development of clinical skills; for example, clinical meetings, courses, lectures or seminars, special attachments at another department or hospital, or sitting in on a colleague's clinic. Academic CPD includes clinical audit, research activities, preparation of postgraduate lectures, postgraduate examining, publishing, presenting audit and research findings, and teaching-the-teacher courses. A period of sabbatical leave, or a postgraduate degree or diploma course might be included in this category, as might writing or revising evidence based service protocols. Professional activities that enhance the ability to practise, and which relate to employers, colleagues, and patients include management, information technology, communication skills and equal opportunities training, medico-legal seminars, and attending Confidential Enquiries into Maternal and Child Health (CEMACH) case reviews.

There are numerous courses and apprenticeship activities that may qualify as external or internal CPD, according to definitions. These

Abbreviations: $C P D$, continuing professional development; PDP, professional development plan; $\mathrm{RCPCH}$, Royal College of Paediatrics and Child Health 
include structured postgraduate courses (masters, diploma, and certificate programmes). The RCPCH is keen to encourage apprenticeship, distance learning (internet, video links, CD-ROMs, and audio tapes) and critical reading groups (for example, journal clubs). The broad nature of the scheme allows recognition of the preparation of examinations, the writing of books, and editorial activities.

The RCPCH (in line with other College schemes) specifies that the basic unit of CPD activity will be one hour (one credit). There are limits set on the number of points that may be claimed in a single category. Paediatricians should allocate themselves points-usually one per hour, though occasionally it may be more appropriate to claim less, according to how much value is received from an educational activity.

\section{PERSONAL RESPONSIBILITY FOR CPD}

The RCPCH audits a random sample of $5 \%$ of the records yearly. This requires individual doctors to send in sufficient documents to verify activities attended. It is therefore important to retain evidence of learning (for example, notes), as well as participation, (for example, programmes, receipts, publications, etc). Paediatricians are required to accrue approximately $250 \mathrm{CPD}$ credits for each five year period on a rolling basis-that is, between 1 January of the first year and 31 December of the fifth year (that is, a target of 50 credits per year). The RCPCH publishes on its website a list of those paediatricians who are participating in the scheme. Participation in the scheme is a prerequisite of being "in good standing" ${ }^{\prime 6}$ with the College.

\section{GENERAL ADVICE}

The most important step is to plan ahead. Acquisition of CPD should not be retrospective.

Almost all questions about CPD can be answered by "reading the manual" (see www.rcpch.ac.uk). Numerous inquiries are received by the CPD office each year. Most of these are covered in the annual guidelines, in which answers to past queries have been incorporated.

Scheme participants should ensure a balanced CPD programme of learning experiences. It is good practice to enter all details on the CPD record monthly at least, if not as they happen. Participants should make returns to the RCPCH office on the postcards provided or through the College website (www.cpd.rcpch.ac.uk), indicating total credits obtained in each of the CPD categories. The record and the accompanying evidence will also help paediatricians to construct a personal development plan (PDP), which should include detailed plans for the forthcoming year's external CPD points acquisition. These may of course be valuable in discussions with clinical director, medical director, and the employer in planning and resourcing study leave.

The requirements for CPD should be part of job descriptions and contracts of employment. The focus of CPD will also vary between paediatricians according to their individual needs and learning styles. For example, the needs of paediatricians working in district general hospitals or the community might differ from those in teaching centres. Those chiefly in academic practice will also be likely to require different types of CPD. All doctors should have an individual plan for their education and professional development. The PDP should help paediatricians think about their learning needs in the context of the needs of the department and Trust. This will help to set targets for CPD and a timetable for their achievement. Finding about one's personal preferred learning style (for example, using Honey and Mumford's profile of pragmatist, theorist, activist, and reflector $^{7}$ ) can also be useful in planning exactly how the next year's credits will best be acquired.

\section{INNOVATIVE APPROACHES AND EXAMPLES OF PRACTICAL SOLUTIONS TO CPD PROBLEMS}

The RCPCH encourages a wide variety of approaches to address difficulties experienced by individuals or groups.

Reflective Notes are an innovative feature of the CPD process. These can make powerful contributions to learning. Reflective Notes presented in the format of the proforma in the RCPCH CPD guidelines, ${ }^{4}$ provide considerable latitude for scheme participants in claiming up to $20 \mathrm{CPD}$ points per annum. The proforma allows recording of a title or description of the experience, what happened, was done, or learnt, and whether the experience highlighted any further learning needs. One credit can be claimed for each completed page of Reflective Notes, identified according to the category (clinical, academic, or professional) considered most appropriate. Reflective Notes may vary from brief accounts to more detailed descriptions of learning.

Activities that qualify for Reflective Notes are diverse; for example, private reading that changes perception, an informal discussion with a colleague that leads to reflection on a clinical problem, a patient encounter, or a critical incident may all be the subject of Reflective Notes. While there are a number of activities that do not directly qualify for CPD credits, writing apposite Reflective Notes on them can do so. Examples include undergraduate teaching and undergraduate examining, domiciliary consultations and reading journals, chairing or attending committees, or performing day-to-day management tasks. If learning has occurred, then recording this as a Reflective Note would be acceptable.

Apprenticeship activities for individuals wishing to learn new practical techniques or to update clinical skills (for example, endoscopy, management of epilepsy), can be recognised. Learning from colleagues in inpatient, outpatient, or community situations, or personal practice sessions may also be claimed as CPD: joint clinics with visiting tertiary specialists often lead to learning points for all career staff who attend, including the specialist. Reflective Notes, listed learning points or (anonymised) patient letters could all act as evidence.

Self-directed learning activities may feature in claims for internal and external CPD (depending on the degree of interaction with colleagues from afar). These could include prepared teaching packages (for example, IT training), distance learning courses, and formal instructing on life support courses. Claims can be made for writing articles which have been peer reviewed and accepted for publication (though not for papers in progress) and for writing and revising literature informed evidence based service protocols.

\section{DOCTORS WHO HAVE DIFFICULTY ACQUIRING CPD}

In exceptional circumstances meeting CPD requirements will be difficult. The RCPCH guidelines can be used to help paediatricians set objectives at annual appraisal, which emphasise their need to achieve their CPD targets.

Several groupings of paediatricians may experience particular difficulties. Some paediatricians may have too little time, or insufficient structure for regular continuing education. These issues may, in turn, be influenced by other demands on time-especially clinical and committee work, which need managing.

External CPD is usually accessed through existing study leave allowances. Paediatricians who work in smaller units, and community doctors, may have limited study leave budgets or problems in finding cover for their duties. Some paediatricians work in relatively isolated areas and may find it especially difficult to acquire external CPD points, for which they need to interact and communicate with colleagues. Employers in these units should be prepared to ensure that suitable study leave funding and locum cover 


\section{Ten top tips to tame your CPD}

- Participate in the RCPCH CPD scheme

- Read the guidelines thoroughly

- Construct a personal development plan

- Plan your forthcoming year's CPD

- Ensure that all aspects of your career are developed

- Consider how you can amass "evidence of learning"

- Collect certificates and copies of all evidence in one file as you go

- Keep a Reflective Note proforma with you at all times

- Write Reflective Notes on what you learn during internal CPD

- Cite CPD scheme guidance to gain funding for external CPD

arrangements are made to enable their career grade staff to acquire external CPD. Employers are already aware that CPD is a professional obligation for updating skills and knowledge and that deficiencies in CPD have serious implications for doctors' appraisals and revalidation and a department's risk management and clinical governance.

The CPD status of all career grade staff in a directorate is monitored when training posts are inspected. Training status cannot reasonably be granted to trainers who are not participating in CPD, preferably on the RCPCH scheme.

The GMC and most Colleges agree that all doctors need to achieve the same minimum 250 CPD points in five yearsapproximately 1 point per week. Part-time paediatricians may experience difficulties in attending CPD activities that are scheduled outside the sessions in which they work. Conversely, employers may be reluctant to allow external CPD activity during a reduced working week. In practice, all parties in this dilemma must be prepared to display some flexibility. External CPD courses will not always coincide with paediatricians' working hours, and employers should not expect part-time paediatricians to undertake all external CPD outside scheduled sessional commitments.

Some paediatricians may experience prolonged absence from work. There are a number of reasons, particularly long term illness, maternity leave, and unusual domestic commitments. In these circumstances time allocated for individuals to meet CPD targets may be extended or the balance between internal and external CPD altered. However, any difficulties or imbalance in one year must be redressed later if GMC revalidation is to proceed every five years.

Reassuringly, evidence from the annual audit suggests that most paediatricians are able to plan and acquire a minimum of 25 external CPD points each year. Sometimes paediatricians in demonstrably difficult circumstances can acquire such points or even an excess. Paediatricians experiencing difficulties should seek the advice and support of RCPCH regional advisers, college tutors, or the CPD office.

\section{CONCLUSION}

Paediatricians are a diverse group who have a wide range of learning needs. Most can use the RCPCH CPD scheme to plan and optimise their CPD. Careful reading of the CPD scheme guidance can help solve problems for those with difficulties acquiring credits.

\section{ACKNOWLEDGEMENTS}

I am most grateful for preliminary discussions and comments on the manuscript from members of the RCPCH CPD Sub-Committee.

Competing interests: none

\section{REFERENCES}

1 General Medical Council. Licensing and revalidation. September 2004. Available at http://www.gmc-uk.org/revalidation/archive/ I_and_r_formal_guidance_for_docs.pdf.

2 Thomson APJ, RCPCH CPD Department. CPD adherence: the first two years of RCPCH CPD audit. Proceedings of Association of Medical Education in Europe (AMEE) Annual Meeting, Amsterdam 1-3 September 2005. Available at http://www.amee.org/.

3 GMC. A licence to practise and revalidation. Available at http:// www.appraisaluk.info o www.revalidationuk.info.

4 Royal College of Paediatrics and Child Health. Continuing professional development for career grade paediatricians - the national scheme of the Academic Board, Royal College of Paediatrics and Child Health, January 2005. London: Royal College of Paediatrics and Child Health, 2005. Available at http://www.rcpch.ac.uk/.

5 Academy of Medical Royal Colleges. Principles of continuing professional development. London: Academy of Medical Royal Colleges, 1999. Available at http://www.aomrc.org.uk/.

6 Royal College of Paediatrics and Child Health. "In good standing" with the RCPCH. London: Royal College of Paediatrics and Child Health, 2004. Available at http://www.rcpch.ac.uk/.

7 Honey P, Mumford A. Learning style questionnaire, 2005. Available at http://www.peterhoney.co.uk/. 